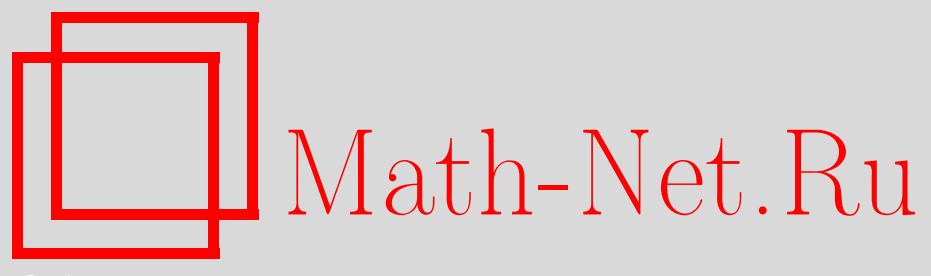

А. Н. Печень, Н. Б. Ильин, О критических точках целевого функционала в задаче максимизации наблюдаемых кубита, УМН, 2015, том 70, выпуск 4, 211-212

DOI: https://doi.org/10.4213/rm9663

Использование Общероссийского математического портала Math-Net.Ru подразумевает, что вы прочитали и согласны с пользовательским соглашением http://www . mathnet.ru/rus/agreement

Параметры загрузки:

IP : 52.90 .164 .192

26 апреля 2023 г., 13:19:02

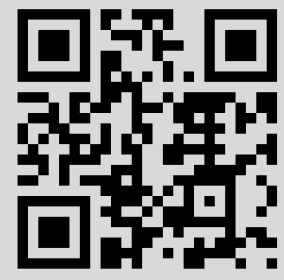




\section{О критических точках целевого функционала в задаче максимизации наблюдаемых кубита}

\section{А. Н. Печень, Н. Б. Ильин}

Изолированная от окружения $n$-уровневая управляемая квантовая система описывается уравнением Шрёдингера для унитарного оператора эволюции $U_{t}$ :

$$
i \frac{d U_{t}}{d t}=\left(H_{0}+f(t) V\right) U_{t}, \quad U_{t=0}=\mathbb{I} .
$$

Здесь $H_{0}$ и $V$ - эрмитовы $(n \times n)$-матрицы и $f(t) \in L^{1}([0, T] ; \mathbb{R})$ - управление. Предполагается, что $\left[H_{0}, V\right] \neq 0$. Требуется найти $f(t)$, которая максимизирует целевой функционал $\mathscr{J}_{A}[f]=\operatorname{Tr}\left(U_{T} \rho_{0} U_{T}^{\dagger} A\right)$, где $\rho_{0}-$ начальная матрица плотности системы, $A$ - эрмитова матрица и $T>0$ - конечное время. Функционал $\mathscr{J}_{A}$ описывает среднее значение наблюдаемой $A$ в момент времени $T$. Важной проблемой является анализ локальных максимумов целевого функционала, называемых ловушками [1]-[3], так как ловушки, если они существуют, затрудняют поиск глобально оптимальных решений. В работах [1], [2] высказано предположение об отсутствии ловушек в типичных задачах управления квантовыми системами. В работах [4], [5] доказано отсутствие ловушек для двухуровневых квантовых систем при достаточно больших $T$. В работе [5] доказано следующее утверждение.

Teоpema 1. Если $\operatorname{Tr} V=0$ u $T \geqslant T_{0}$, где $T_{0}=\pi /\left\|H_{0}-(1 / 2) \operatorname{Tr} H_{0}+f_{0} V\right\| u$ $f_{0}=-\operatorname{Tr}\left(H_{0} V\right) / \operatorname{Tr}\left(V^{2}\right)$, то все максимумы целевого функционала $\mathscr{J}_{A}$ - глобальные.

Из результатов работы [5] следует, что никакое управление $f \neq f_{0}$ не является ловушкой для любого $T>0$. Таким образом, только управление $f=f_{0}$ может быть ловушкой при малых $T$.

Далее рассматривается частный случай уравнения Шрёдингера (1) вида:

$$
i \frac{d U_{t}}{d t}=\left(\sigma_{z}+f(t)\left(v_{x} \sigma_{x}+v_{y} \sigma_{y}\right)\right) U_{t}
$$

Здесь $\sigma_{x}, \sigma_{y}, \sigma_{z}$ - матрицы Паули. В этом случае $f_{0}=0$ и $T_{0}=\pi$. В [6] доказано, что ловушки в рассматриваемой системе возможны при малых $T$, но только если векторы $\mathbf{r}^{0}=\operatorname{Tr}\left(\rho_{0} \boldsymbol{\sigma}\right), \mathbf{a}=\operatorname{Tr}(A \boldsymbol{\sigma})$ и $\mathbf{v}=(1 / 2) \operatorname{Tr}(V \boldsymbol{\sigma})\left(\boldsymbol{\sigma}=\left(\sigma_{x}, \sigma_{y}, \sigma_{z}\right)\right)$ лежат в плоскости, ортогональной вектору $\mathbf{h}_{0}=\operatorname{Tr}\left(H_{0} \boldsymbol{\sigma}\right)$. В работе [6] доказан следующий результат.

TeOpema 2. Пусть $\left[\left(\mathbf{v} \times \mathbf{r}^{0}\right)_{z} \cos 2 T+\left(\mathbf{v} \cdot \mathbf{r}^{0}\right) \sin 2 T\right](\mathbf{v} \times \mathbf{a})_{z}>0 u\left(\mathbf{r}^{0} \times \mathbf{a}\right)_{z} \cos 2 T<$ $\left(\mathbf{r}^{0} \cdot \mathbf{a}\right) \sin 2 T$, а векторы $\mathbf{r}^{0}, \mathbf{a} u \mathbf{v}$ лежат в плоскости, ортогональной вектору $\mathbf{h}_{0}$. Тогда существует такое $T_{1}$, что для всех $T \leqslant T_{1}$ управление $f(t)=0$ является ловушкой для задачи максимизации целевого функционала $\mathscr{J}_{A}$.

Несмотря на этот результат, проблема анализа ловушек для двухуровневых систем не была решена до конца. Так, для системы (2) согласно теореме 1 ловушки отсутствуют для всех $T \geqslant T_{0}=\pi$ и согласно теореме 2 ловушки существуют для всех $T \leqslant T_{1}$, причем величина $T_{1}$ не конкретизируется. Ниже мы доказываем, что нижнюю границу на $T$ для отсутствия ловушек можно уменьшить.

Теорема 3. При $T>\pi / 2$ управление $f=0$ не является ловушкой в задаче максимизачии иелевого функиионала $\mathscr{J}_{A}$ для системъ (2).

Исследование выполнено за счет гранта Российского научного фонда (проект № 14-50-00005). DOI: $10.4213 / \mathrm{rm} 9663$ 
Для того чтобы доказать, что управление $f=0$ не ловушка, покажем, что если $f=0$ - критическая точка, то это либо глобальный экстремум, либо седловая точка. В последнем случае достаточно убедиться, что гессиан в точке $f=0$ не знакоопределен. В работе [6] доказано, что управление $f=0$ может оказаться ловушкой, только если векторы $\mathbf{r}^{0}, \mathbf{a}$ и $\mathbf{v}$ лежат в плоскости, ортогональной вектору $\mathbf{h}_{0}$. При этом условии на векторы $\mathbf{r}^{0}, \mathbf{a}$ и $\mathbf{v}$ в [5] получено выражение для гессиана в точке $f=0$ через векторы $\mathbf{r}=\operatorname{Tr}\left(U_{T} \rho U_{T}^{\dagger} \boldsymbol{\sigma}\right)$ и $\mathbf{r}_{t}=\sin (2 t-\phi) \mathbf{e}_{x}+\cos (2 t-\phi) \mathbf{e}_{y}$, где $\phi=\arctan \left(v_{y} / v_{x}\right)$ :

$$
\begin{aligned}
(f, H f) & =\int_{0}^{T} \int_{0}^{T} f\left(t_{1}\right) f\left(t_{2}\right) \operatorname{Hess}_{f} \mathscr{J}_{A}\left(t_{2}, t_{1}\right) d t_{1} d t_{2}, \\
\operatorname{Hess}_{f} \mathscr{J}_{A}\left(t_{2}, t_{1}\right) & = \begin{cases}-\frac{v^{2}}{4}\left(\mathbf{r} \cdot \mathbf{r}_{t_{2}}\right)\left(\mathbf{a} \cdot \mathbf{r}_{t_{1}}\right), & t_{2} \geqslant t_{1}, \\
-\frac{v^{2}}{4}\left(\mathbf{r} \cdot \mathbf{r}_{t_{1}}\right)\left(\mathbf{a} \cdot \mathbf{r}_{t_{2}}\right), & t_{2}<t_{1} .\end{cases}
\end{aligned}
$$

Рассмотрим вспомогательную функцию

$$
\delta_{\varepsilon}(t)= \begin{cases}0, & |t| \geqslant \varepsilon / 2, \\ 1 / \varepsilon, & |t| \leqslant \varepsilon / 2\end{cases}
$$

Тогда для всех $f \in \mathrm{C}[0, T]$ и всех $t \in(\varepsilon / 2, T-\varepsilon / 2), \varepsilon<T$, имеем $\int_{0}^{T} \delta_{\varepsilon}(\tau-t) f(\tau) d \tau=$ $f(t)+O(\varepsilon)$. Пусть $f_{\lambda}(t)=\delta_{\varepsilon}(t-\lambda), \varepsilon / 2<\lambda<T-\varepsilon / 2$. Подставляя функцию $f_{\lambda}(t)$ в выражение для гессиана (3), получим $\left(f_{\lambda}, H f_{\lambda}\right)=-\left(v^{2} / 4\right)\left(\mathbf{r} \cdot \mathbf{r}_{\lambda}\right)\left(\mathbf{a} \cdot \mathbf{r}_{\lambda}\right)+O(\varepsilon)$, где

$$
\left(\mathbf{r} \cdot \mathbf{r}_{\lambda}\right)\left(\mathbf{a} \cdot \mathbf{r}_{\lambda}\right)=\frac{|\mathbf{r}||\mathbf{a}|}{2}\left(\cos \left(\phi_{1}-\phi_{2}\right)-\cos \left(4 \lambda-2 \phi+\phi_{1}+\phi_{2}\right)\right) .
$$

Здесь $\phi_{1}=\arctan \left(r_{y} / r_{x}\right), \phi_{2}=\arctan \left(a_{y} / a_{x}\right)$. Если $\cos \left(\phi_{1}-\phi_{2}\right) \neq \pm 1$, то из формулы (5) вытекает, что функция $\left(\mathbf{r} \cdot \mathbf{r}_{\lambda}\right)\left(\mathbf{a} \cdot \mathbf{r}_{\lambda}\right)$ принимает значения разных знаков на отрезке $[0, \pi / 2]$. В этом случае выбираем $\lambda_{1}$ и $\lambda_{2}$ так, чтобы $\left(\mathbf{r} \cdot \mathbf{r}_{\lambda_{1}}\right)\left(\mathbf{a} \cdot \mathbf{r}_{\lambda_{1}}\right)<0$ и $\left(\mathbf{r} \cdot \mathbf{r}_{\lambda_{2}}\right)\left(\mathbf{a} \cdot \mathbf{r}_{\lambda_{2}}\right)>0$, и затем выбираем $\varepsilon$ настолько малым, чтобы знак $\left(f_{\lambda}, H f_{\lambda}\right)$ определялся знаком $\left(\mathbf{r} \cdot \mathbf{r}_{\lambda}\right)\left(\mathbf{a} \cdot \mathbf{r}_{\lambda}\right)$. Тогда $\left(f_{\lambda_{1}}, H f_{\lambda_{1}}\right)>0$ и $\left(f_{\lambda_{2}}, H f_{\lambda_{2}}\right)<0$, т. е. гессиан в точке $f=0$ не является знакоопределенной функцией. В этом случае управление $f=0$ является седловой точкой. Если же $\cos \left(\phi_{1}-\phi_{2}\right)= \pm 1$, то векторы $\mathbf{r}$ и а параллельны. Тогда $\mathscr{J}_{A}[0]=(1 \pm|\mathbf{a}||\mathbf{r}|) / 2$, что является глобальным экстремумом целевого функционала.

\section{Список литературы}

[1] H. A. Rabitz, M. M. Hsieh, C. M. Rosenthal, Science, 303:5666 (2004), 1998-2001. [2] T.-S. Ho, H. Rabitz, J. Photochem. Photobiol. A, 180:3 (2006), 226-240. [3] P. de Fouquieres, S. G. Schirmer, Infin. Dimens. Anal. Quantum Probab. Relat. Top., 16:3 (2013), 1350021. [4] A. Pechen, N. Il'in, Phys. Rev. A, 86:5 (2012), 052117. [5] А.Н. Печень, Н. Б. Ильин, Тр. МИАН, 285, 2014, 244-252. [6] А. Печень, Н. Ильин, Тр. МИАН, 289, 2015, 227-234.

А. Н. Печень (А. N. Pechen)

Математический институт им. В. А. Стеклова

Российской академии наук

E-mail: pechen@mi.ras.ru

Н. Б. Ильин (N. В. Il'in)

Математический институт им. В. А. Стеклова

Российской академии наук

E-mail: ilyn@mi.ras.ru
Представлено А. Г. Сергеевым Принято редколлегией 10.04.2015 